\title{
Effect of co-payment on dental visits: A regression discontinuity analysis
}

Upul Cooray (MSc) $)^{1,2}$, Jun Aida $(\mathrm{PhD})^{1}$, Richard G Watt $(\mathrm{PhD})^{2}$, Georgios Tsakos $(\mathrm{PhD})^{2}$, Anja Heilmann $(\mathrm{PhD})^{2}$, Hirotaka Kato $(\mathrm{PhD})^{3}$, Sakura Kiuchi (DDS) ${ }^{1}$, Katsunori Kondo (PhD) ${ }^{4,5}$, Ken Osaka $(\mathrm{PhD})^{1}$

${ }^{1}$ Department of International and Community Oral Health, Tohoku University Graduate School of Dentistry, Sendai, Japan

${ }^{2}$ Department of Epidemiology and Public Health, University College London, London, United Kingdom

${ }^{3}$ Graduate School of Business Administration, Keio University, Yokohama, Japan

${ }^{4}$ Center for Preventive Medical Sciences, Chiba University, Chiba, Japan

${ }^{5}$ Center for Gerontology and Social Science, National Center for Geriatrics and Gerontology, Obu, Japan

\section{Corresponding Author:}

Jun Aida

Department of International and Community Oral Health, Tohoku University Graduate School of Dentistry, Sendai, Japan

Address: 4-1, Seiryo-machi, Aoba-ku, Sendai, Miyagi 980-8575, Japan

Telephone: +81 22-717-7639

E-mail: j-aida@umin.ac.jp

Abstract word count: 292

Total word count: 3198

Number of tables: 2

Number of figures: 2

Number of reference: 34

Keywords: Dental public health, Health services research, Epidemiology, Behavioural Science, Access to care, Prevention 


\section{Effect of co-payment on dental visits: A regression discontinuity analysis}

Upul Cooray $^{1,2}$, Jun Aida ${ }^{1}$, Richard G Watt ${ }^{2}$, Georgios Tsakos ${ }^{2}$, Anja Heilmann ${ }^{2}$, Hirotaka Kato ${ }^{3}$ Sakura Kiuchi ${ }^{1}$, Katsunori Kondo ${ }^{4,5}$, Ken Osaka ${ }^{1}$

${ }^{1}$ Department of International and Community Oral Health, Tohoku University Graduate School of Dentistry, Sendai, Japan

${ }^{2}$ Department of Epidemiology and Public Health, University College London, London, United Kingdom

${ }^{3}$ Graduate School of Business Administration, Keio University, Yokohama, Japan

${ }^{4}$ Center for Preventive Medical Sciences, Chiba University, Chiba, Japan

${ }^{5}$ Center for Gerontology and Social Science, National Center for Geriatrics and Gerontology, Obu, Japan 


\begin{abstract}
Despite their prevalence and burdens, oral diseases are neglected in universal health coverage (UHC). In Japan, a 30\% co-payment (out-of-pocket) by the user, and a $70 \%$ contribution by the Universal Healthcare Insurance (JUHI) are required for dental and medical services. From the age of 70, an additional $10 \%$ is offered by JUHI (co-payment - 20\%, JUHI - $80 \%$ ). This study aimed to investigate the effect of cost on dental services use among older adults under the current JUHI system. Regression discontinuity (RD) quasi-experimental method was used to investigate the causal effect of the JUHI discount policy on dental visits using cross-sectional data. Data were derived from the 2016 Japanese Gerontological Evaluation Study (JAGES 2016). This analysis contained 7,161 participants using JUHI and aged 68-73 years who responded to questions regarding past dental visits. Analyses were controlled for age, sex, number of teeth, and equalised household income. Mean age was 72.1 ( $\mathrm{SD} \pm 0.79$ ) years for the discount eligible group and 68.9 ( $\mathrm{SD} \pm 0.78$ ) years for the non-eligible group. During the past 12 months, significantly more discount eligible participants had visited dental services than non-eligible participants $(66.0 \%$ vs. $62.1 \%$ for treatment visits, $57.7 \%$ vs. $53.1 \%$ for check-ups). After controlling for covariates the effect of discount eligibility was significant on both dental treatment visits and dental check-ups $(\mathrm{OR}=1.36,95 \% \mathrm{CI} 1.32-1.40$ and $\mathrm{OR}=1.49,95 \% \mathrm{CI} 1.44-$ 1.54 , respectively) in the RD analysis. Similar findings were observed in triangular kernel weighted models $(\mathrm{OR}=1.38,95 \% \mathrm{CI} 1.34-1.44$ and $\mathrm{OR}=1.52$, 95\%CI 1.47-1.56, respectively). JUHI copayment discount policy increases oral health services utilisation among older Japanese. The price elasticity for dental check-up visits appear to be higher than for dental treatment visits. Hence, reforming the UHC system to improve the affordability of relatively inexpensive preventive care could further increase dental service utilisation in Japan.
\end{abstract}




\section{Introduction}

The Global Burden of Diseases Study 2017 revealed that oral disorders such as dental decay and periodontal diseases remained highly prevalent global disorders since 1990 (James et al. 2018). Oral diseases affect individuals' quality of life by causing pain, discomfort, and inability to enjoy food (Sheiham and Steele 2001; Griffin et al. 2012). Moreover, the high prevalence of oral diseases causes substantial economic and societal burden due to high treatment costs and disability (Birch and Listl 2015; Bernabé et al. 2017). As with chronic conditions in general, the impact of oral diseases is higher on poorer and less educated populations of society (Watt et al. 2016; Rozier et al. 2017). Universal Health Coverage (UHC) is put forward as a global policy to enable all individuals and communities irrespective of their socioeconomic position, to receive health services without suffering financial hardship (WHO 2005). However, in many countries, oral health is usually isolated from mainstream primary healthcare services and neglected in UHC (Watt et al. 2019).

According to WHO, countries can achieve greater UHC along three dimensions - by extending population coverage, by including services not previously covered, and by reducing cost sharing and fees (WHO 2013). Treatment of oral diseases is costly and often associated with high out of pocket expenditure for service users (Bernabé et al. 2017; Dieleman et al. 2020). Thus, including oral health services in the UHC agenda would be one way of increasing coverage that could potentially minimise cost related inequalities in service use.

Japan has one of the most generous mandatory universal health insurance schemes in the world. Japan's universal health insurance (JUHI) policy is unique as it covers a wide range of dental treatments at a lower cost for its subscribers, compared to other countries with 'universal oral healthcare systems' (Zaitsu et al. 2018). Among OECD countries only Japan (78\%), Germany (68\%), and Slovak Republic (53\%) cover more than half of the dental care cost of adults (OECD 2019). Furthermore, Japan had reported the most frequent dental visits among OECD countries (OECD 2011). In 2013, the prevalence of edentulousness among Japanese adults aged 65-74 years was 6.9\%, which compares to an average of $13.3 \%$ among 15 European countries (Stock et al. 2016).However, inequalities in access to dental services still exist in Japan (Murakami et al. 2014). Therefore, assessing the cost sensitivity of services utilisation in a country where oral health is already a part of the UHC system could provide a basis for comparisons with cost effects in the absence of a UHC system for oral health.

In general, the use of dental and medical services in Japan requires a 30\% co-payment (out-of-pocket) by the user, and a $70 \%$ contribution by the JUHI. When JUHI subscribers reach the age of 70 , an additional $10 \%$ is offered, starting from the month after their birth month (JUHI - 80\%, co-payment 
- 20\%), which is a $33 \%$ reduction in co-payment (Sakamoto et al. 2018). This policy rule provides a unique opportunity to use the regression discontinuity (RD) method to investigate the causal effect of changes in out-of-pocket cost on dental attendance in Japan. The RD method is able to capture an observable discontinuity in a treatment when the level of treatment changes abruptly at a cut-off value of treatment assignment variable (Imbens and Lemieux 2008).

Using RD, Shigeoka (2014) and Fukushima et al. (2016) found that the reduction of co-payments at the age of 70 increased the utilisation of inpatient and outpatient medical services in Japan. Furthermore, Fukushima et al. (2016) found that the price responsiveness was high for services which are at high demand in an aging society, such as orthopaedic and eye specialities. Although dental treatments are at high demand among older adults, neither of these studies investigated the utilisation of dental services. Ando and Takaku (2016) used a similar methodology to show the effect of copayment reduction on denture use and subjective chewing ability. However, the data were prior to the implementation of the implementation of current co-payments rates in 2014 and they did not examine the impact of co-payment on dental attendance.

The aim of this study was to investigate whether the use of oral healthcare services differed before and after the discount JUHI eligibility, and if a significant difference was observed, to investigate the causal effect of the JUHI discount policy on the observed difference.

\section{Methods}

\section{Data Source}

Our analyses are based on cross-sectional data from the Japan Gerontological Evaluation Study (JAGES) in 2016 (Kondo et al. 2018). The target population of the JAGES survey were communitydwelling older people aged 65 years or older who were functionally independent. The JAGES survey was conducted from September 2016 to November 2017 and collected information on health, behaviours, and socio-economic characteristics via a postal survey questionnaire.

The target population for JAGES in 2016 was 279,661 potential participants, of which 180,021 people responded. Around $1 / 8$ of the target population (34,567 people) received the questionnaire containing questions regarding dental visits, and 22,282 people responded. Respondents with missing values for outcome variables were not included in analyses. Characteristics of the participants with missing information for outcomes were compared and reported in Appendix Table 1. Observations with missing values for other covariates were imputed using multiple imputation (Appendix Table 2). 
Participants who received social welfare benefits were excluded as they were eligible for free dental care without a co-payment. Figure 1 shows the selection flow of participants in this study.

Ethical approval

The JAGES survey was approved by the ethics committee of the National Center for Geriatrics and Gerontology (No.992) and the ethics committee of Chiba University (No.2493).

Outcome variables (Y)

This study used two outcome variables that reflect dental attendance during the past 12 months. Outcome variables were derived from responses to the questions regarding the time of the latest dental treatment visits and dental check-up visits, with response options: 1. during the past 6 months; 2. Six months to 1 year ago; 3 . One to 3 years ago; 4 . More than 3 years ago; 5 . Never seen a dentist. We created dichotomous variables for both treatment visits and check-up visits combining responses 1 and $2=1$ (during past 12 months); 3, 4 and $5=0$ (more than 12 months ago).

Forcing variable $(\mathrm{Z})$

The forcing variable was used to assign observations into 'treatment' and 'control' groups around a cut-off value of the forcing variable (Imbens and Lemieux 2008). The co-payment rate for dental care was $30 \%$ among those aged 69 years or younger and $20 \%$ among those aged 70 years or older. We used age as the forcing variable $(\mathrm{Z})$, with age 71 years as the cut-off point. 71 years cut-off point was chosen as the outcome variables were referring to dental visits during the past 12 months. Hence, those who were 70 years at the time of survey were included in the control group $(68,69$, 70 as 'noneligible' and 71, 72, 73 as 'discount eligible'). People who turned 70 before 2014 continued to receive a co-payment discount under the old co-payment scheme (which was 33\% generous than current rate). Therefore, we limited the maximum age as less than 74 years at the time of the survey to avoid the inclusion of participants falling under the old co-payment scheme. A derived age variable centered on the cut-off value (age 71 years $=0$ ) was used in analyses.

Treatment variable/ discount eligibility (T)

A dummy treatment assignment variable $(\mathrm{T})$ was created using the age cut-off; $\mathrm{T}=0$ if $\mathrm{Z}<$ cut-off, and $\mathrm{T}=1$ if $\mathrm{Z} \geq$ cut-off. This variable estimated the effect of discount eligibility in RD models.

Covariates $(\mathrm{X})$

A categorical variable indicating the number of remaining teeth $(0,1-9,10-19$, more than 20 teeth) and equivalised annual household income in Japanese yen $(<2$ million, $>=2$ to $<3$ million, $>=3$ to $<4$ 
million, $>=4$ million) were used as covariates in the analyses. Equivalised income was calculated by dividing annual household income by the square root of number of family members. Furthermore, all models were adjusted for sex, and age (forcing variable).

Statistical analysis

Descriptive analysis presented the characteristics of the participants against discount eligibility. Then we adopted a regression discontinuity approach to estimate the effect of JUHI discount eligibility on dental attendance during the past 12 months. Following the methodology suggested by Jacob et al. (2012), we tested a variety of functional forms of the forcing variable (Z) to identify which fitted our data best based on F statistics and the robustness of estimates (Appendix Table 3 and 4). We used both linear and quadratic interaction forms of the forcing variable. In line with recommendations (Gelman and Imbens, 2019), we avoided higher order polynomials as it can lead to nonsensical results. The following logistic regression model was used to estimate the effect of discount eligibility on the probability of dental attendance during the past 12 months.

$\mathrm{E}[\mathrm{Y} i \mid \mathrm{T} i, \mathrm{Z} i, \mathrm{X} i]=\exp \left[\beta 0+\beta 1 \mathrm{~T} i+\beta 2 \mathrm{Z} i+\beta 3 \mathrm{~T} i * \mathrm{Z} i+\beta 4 \mathrm{Z} i+\beta 5 f i^{*} \mathrm{~T}+\beta 6 \mathrm{X} i+\varepsilon\right]$

$\mathrm{Y}=$ Probability of a dental visit during past 12 months

$\mathrm{T}=$ Dummy treatment variable ( 1 if $\mathrm{Z} \geq$ cut-off, 0 if $Z<$ cut-off)

$Z=$ Forcing variable (age-centered variable)

$f=$ polynomial function of $Z$ (quadratic function)

$X=$ Matrix of covariates (number of teeth, income, sex)

$\beta 1=$ Measure the impact of discount eligibility (RD estimate)

$\varepsilon=$ Error term

Logistic regression models were predicting the likelihood of dental attendance during the past 12 months for dental treatments and for dental check-ups. To check the robustness of RD estimate, a triangular kernel weighted model, which assign more weight to observations closer to the cut-off was used. We used 20 multiple imputed datasets for RD analyses. Then, a single mean estimate and adjusted standard errors were calculated (Rubin 2004). A supplementary complete case only analysis assess the robustness of imputed analysis (Appendix Table 5).

Stata MP version 15.1 was used for analyses. RD plots were constructed using matplotlib python library. The reporting of this study conforms to STROBE guidelines.

\section{Results}

Table 1 reports descriptive statistics on outcome variables and covariates, by discount eligibility. The mean age of discount eligible group was 72.1 ( $\mathrm{SD} \pm 0.79$ ) years and $68.9(\mathrm{SD} \pm 0.78$ ) years for the noneligible group. Discount eligible participants reported higher prevalence of dental treatment and 
check-up visits, than non-eligible participants during the past 12 months (treatment visits- $66.0 \%$ vs. $62.1 \%$; $<<0.001$, check-up visits- $57.7 \%$ vs. $53.1 \%$; $\mathrm{p}<0.001)$. The discount eligible group had higher proportions of edentulous and people in the lowest household income compared to the non-eligible group.

RD estimates and other covariates related coefficients from unweighted and triangular kernel weighted logistic models are reported as odds ratios (OR) in Table 2. Coefficients related to the dummy variable that indicates discount eligibility $(\mathrm{T})$ measured the change in the probability of dental attendance during the past 12 months between discount eligible and non-eligible groups, after adjusting for covariates. In both unweighted and weighted models the discount eligibility (RD estimate) was associated with significantly higher prevalence of dental treatment visits and dental check-up visits during the past 12 months. In the unweighted models discount eligibility increased the odds of treatment and check-up visits in the past 12 months with ORs of 1.36 (95\%CI 1.32-1.40) and 1.49 (95\%CI 1.44-1.54), respectively. The effect of discount eligibility appeared to be slightly higher in triangular weighted models (Table 2). Being female and having more teeth were strong predictors of treatment visits and check-up visits, irrespective of the effect of discount eligibility. The highest income group was more likely to have attended check-up visits during past 12 months compared to the lowest income group (unweighted model: $\mathrm{OR}=1.18,95 \% \mathrm{CI} 1.03-1.34$ and weighted model: $\mathrm{OR}=1.13,95 \% \mathrm{CI} 0.98-1.30$ ).

Means of predicted probabilities of dental visits during the past 12 months for each age were plotted with second order polynomial fit lines in Figure 2. Plots related to both treatment and check-up visits showed clear discontinuities in the probability of visits during the past 12 months, before and after the discount eligible age cut-off.

\section{Discussion}

To the best of our knowledge, this study is the first to determine the effect of cost burden on dental attendance using a quasi-experimental method. We examined the impact of the JUHI discount eligibility on dental attendance within a population of Japanese adults in order to identify how a costrelated policy could influence UHC in oral health. The findings of our study suggest that a higher proportion of Japanese older adults in the 71-73 year age group used dental services in the last 12 months compared to the 68-70 years old group. RD analysis revealed that the co-payment reduction at the age of 70 significantly contributed towards this higher prevalence of dental service use among older adults aged 71-73 years. Furthermore, the effect of the discount appeared to be slightly higher for dental check-up visits than dental treatment visits. Our findings are consistent with findings of 
Shigeoka (2014) and Fukushima et al. (2016) regarding the effect of co-payment reduction on utilisation of inpatient and outpatient medical services in Japan.

There are several strengths and limitations in this study. To determine the relationship between cost burden and dental attendance, the RD method is one of the best approaches for causal inference as a randomized controlled trial is not feasible (Berk et al. 2010; Leeuwen et al. 2018). The healthcare setting in Japan is uniquely favourable to the use of the RD method as most dental treatments are covered by the universal health care insurance and the amount of co-payments do not vary under this scheme. Hence, we were able to implement the RD method with confidence to isolate the effect of co-payment reduction on dental attendance. The RD method could plausibly account for unmeasured confounders among discount eligible and non-eligible groups as they are assigned by a deterministic function of the age among a group of participants belong to a narrow age range (68-73 years).

A limitation of the study was that our data did not contain birthdays or age in months, therefore we were not able to identify the exact point at which participants became eligible for the discount. Thus, we used the age of 71 at the time of the survey as the cut-off for discount eligibility rather than the actual eligible age of 70, as we were referring to past dental visits in cross sectional data. However, this bias possibly attenuates rather than overestimate the impact of discount eligibility on dental attendance. Although current welfare benefit receivers were excluded from the analyses, the history of welfare benefit receipts was not available in the data and therefore could not be accounted for. Nevertheless, any bias caused by the inclusion of participants with a history of welfare benefit receipt would have underestimated the effect of the co-payment discount, because those who received welfare benefits would not have been expected to change their attendance behaviour based on the discount. Another inherent problem of RD designs related to payment policies is the anticipatory behaviour of participants. That is, when people anticipate a discount in the near future they tend to wait until they become eligible (Figure 2). This anticipatory behaviour might lead to an overestimation of the effect of discount eligibility. However, we believe that the 3 -year bandwidth we applied was long enough to reduce potential bias from anticipatory behaviour relating to dental attendance. In fact, although the effect of bias from anticipatory behaviour could be stronger in the triangular kernel weighted RD model, the difference between unweighted and weighted estimates was small. Finally, we chose not to undertake sex stratified analysis due to the limited sample size and instead performed pooled analyses controlling for sex. Previous research reported that denture utilisation with respect to the reduction of the co-payment in Japan was high for women but close to zero for men (Ando and Takaku 2016). However, our analyses suggested that although women 
utilised significantly more dental services than men, the effect of co-payment reduction was consistent among both sexes, further supporting the aforementioned analytical choice.

Income is one of the most consistent predictors of dental attendance (Listl et al. 2014). In our study, we observed a statistically significant variation in dental check-up visits between the lowest and the highest household income groups. Our findings also suggest that dental check-up visits may be more sensitive to co-payment reduction than treatment visits. Dental treatment visits may be associated with emergency care, whereas dental check-ups are indicative of a more routine pattern of attendance and a more preventive approach. Therefore, treatment visits may be less affected by cost and may have a lower price elasticity than preventive visits. In fact, a previous study in Japan reported that preventive dental visits were more affected by the income of participants than curative treatment (Murakami et al. 2014). These results suggest that higher co-payment could reduce primarily preventive rather than treatment dental visits. These discrete findings might not be generalisable to other countries as dental attendance behaviour would greatly be affected by the country context of the oral health care system. In Japan $78 \%$ of dental care costs were covered by the public insurance (OECD 2019). This percentage was the highest among OECD countries. Therefore, in countries with lower public insurance coverage for dental care, the effect of changes in co-payment would be smaller. However, the cost of services has repeatedly been shown one of the main barriers to dental attendance in many countries (Donaldson et al. 2008; Thompson et al. 2014; Vujicic et al. 2016).

Our findings have clear policy implications. Globally Japan is considered as one of the best countries regarding dental care coverage in UHC, with one of the lowest out-of-pocket dental expenditures (OECD 2011) and most frequent annual dental visits among all OECD countries (OECD.Stat 20102016). This study indicates that dental care access is affected by the level of co-payment even in Japan. Regardless of the good coverage, there are social inequalities in access to dental care in Japan (Murakami et al. 2014; Nishide et al. 2017). Therefore, actions to improve universal coverage for dental care should focus on reducing the cost and tackling underling inequalities. In addition, the present study shows that co-payments disproportionately affect preventive care. Hence, reducing copayments would potentially improve oral health service utilisation and help to re-orient oral healthcare from being 'treatment-oriented' to 'prevention-oriented'. Prevention-oriented oral health UHC system could potentially benefit more people in terms of access to dental services in Japan. However, our study merely investigated if the co-payment reduction was meaningful in terms of dental attendance. While an service related outcome on its own right, dental attendance is one, but clearly not the only, predictor of good oral health in older adults. It is important to further study whether the cost reductions translate into meaningful improvements in oral health. Furthermore, 
future research could investigate the effect of the cost compared to other known predictors of dental attendance such as dental anxiety, attitudes towards oral health, and availability and accessibility of services.

\section{Conclusions}

Older adults in Japan attend dental services in higher proportions after the reduction of co-payments. After controlling for number of teeth, household income, and sex, the co-payment reduction at the age of 70 significantly improved dental attendance. Cost elasticity for preventive dental visits seems to be higher than for treatment visits.

\section{$\underline{\text { Acknowledgments }}$}

The authors gratefully acknowledge the participants in the JAGES 2016 survey. JAGES 2016 was supported by JSPS (Japan Society for the Promotion of Science) KAKENHI Grant Number (JP15H01972), Health Labour Sciences Research Grant (H28-Choju-Ippan-002, H30-Junkankitou-Ippan-004), Japan Agency for Medical Research and Development (AMED) (JP17dk0110017， JP18dk0110027， JP181s0110002， JP18le0110009， JP19dk0110034, JP19dk0110037), Open Innovation Platform with Enterprises, Research Institute and Academia (OPERA, JPMJOP1831) from the Japan Science and Technology (JST), and the Research Funding for Longevity Sciences from National Center for Geriatrics and Gerontology (29-42, 30-22). U. Cooray is supported by Japanese Government (MEXT) Scholarship.

The authors declare no potential conflicts of interest with respect to the authorship and/or publication.

\section{Funding}

The authors received no financial support for the research, authorship, or publication of this article from any funding agency in the public, commercial, or non-profit sectors.

\section{Author contributions:}

U. Cooray: Contributed to conception, design, performed all statistical analyses and interpretation, and drafted the manuscript

J. Aida: Contributed to conception, design, data acquisition and interpretation, critically revised the manuscript

R.G. Watt, G. Tsakos: Contributed to interpretation and critically revised the manuscript

A. Heilmann: Contributed to draft manuscript and critically revised the manuscript 
H. Kato: Contributed to analysis and critically revised the statistical analyses

S. Kiuchi: Contributed to conception and design, and critically revised the manuscript

K. Kondo: Contributed to data acquisition, and critically revised the manuscript

K. Osaka: Contributed to conception and critically revised the manuscript

\section{All authors gave their final approval and agree to be accountable for all aspects of the work.}

All data used are from the JAGES 2016 survey. Data requests can be addressed to the JAGES data management committee via e-mail: dataadmin.ml@jages.net. All JAGES datasets have ethical or legal restrictions for public deposition due to inclusion of sensitive information from human participants.

\section{References}

Ando M, Takaku R. 2016. Affordable false teeth: The effects of patient cost sharing on denture utilization and subjective chewing ability. BE J Econ Anal Policy. 16(3):1387-1438. doi:10.1515/bejeap-2015-0194.

Berk RA, Barnes G, Ahlman L, Kurtz E. 2010. When second best is good enough: A comparison between a true experiment and a regression discontinuity quasi-experiment. J Exp Criminol. 6(2):191-208. doi:10.1007/s11292-010-9095-3.

Bernabé E, Masood M, Vujicic M. 2017. The impact of out-of-pocket payments for dental care on household finances in low and middle income countries. BMC Public Health. 17(1):109. doi:10.1186/s 12889-017-4042-0.

Birch S, Listl S. 2015. The Economics of Oral Health and Health Care. SSRN Electron J.(Max Planck Institute for Social Law and Social Policy Discussion Paper No. 07-2015).

doi:10.2139/ssrn.2611060.

Dieleman JL, Cao J, Chapin A, Chen C, Li Z, Liu A, Horst C, Kaldjian A, Matyasz T, Scott KW, et al. 2020. US Health Care Spending by Payer and Health Condition, 1996-2016. JAMA - J Am Med Assoc. 323(9):863-884. doi:10.1001/jama.2020.0734.

Donaldson AN, Everitt B, Newton T, Steele J, Sherriff M, Bower E. 2008. The effects of social class and dental attendance on oral health. J Dent Res. 87(1):60-64.

doi:10.1177/154405910808700110.

Fukushima K, Mizuoka S, Yamamoto S, Iizuka T. 2016. Patient cost sharing and medical expenditures for the Elderly. J Health Econ. 45:115-130. doi:10.1016/j.jhealeco.2015.10.005.

Gelman A, Imbens G. 2019. Why High-Order Polynomials Should Not Be Used in Regression Discontinuity Designs. J Bus Econ Stat. 37(3):447-456. doi:10.1080/07350015.2017.1366909.

Griffin SO, Jones JA, Brunson D, Griffin PM, Bailey WD. 2012. Burden of oral disease among older adults and implications for public health priorities. Am J Public Health. 102(3):411-418. doi:10.2105/AJPH.2011.300362.

Imbens G, Lemieux T. 2008. Regression Discontinuity Designs: A Guide to Practice. J Econom. 142(2):615-635. doi:https://doi.org/10.1016/j.jeconom.2007.05.001. 
Jacob R, Zhu P, Somers M-A, Bloom H. 2012. A Practical Guide to Regression Discontinuity. [accessed 2020 Mar 27]. www.mdrc.org.

James SL, Abate D, Abate KH, Abay SM, Abbafati C, Abbasi N, Abbastabar H, Abd-Allah F, Abdela J, Abdelalim A, et al. 2018. Global, regional, and national incidence, prevalence, and years lived with disability for 354 Diseases and Injuries for 195 countries and territories, 1990-2017: A systematic analysis for the Global Burden of Disease Study 2017. Lancet. 392(10159):1789-1858. doi:10.1016/S0140-6736(18)32279-7.

Kondo K, Rosenberg M, WHO. 2018. Advancing universal health coverage through knowledge translation for healthy ageing: lessons learnt from the Japan gerontological evaluation study. World Health Organization.

Leeuwen N Van, Lingsma HF, Mooijaart SP, Nieboer D, Trompet S, Steyerberg EW. 2018. Regression discontinuity was a valid design for dichotomous outcomes in three randomized trials. J Clin Epidemiol. 98:70-79. doi:10.1016/j.jclinepi.2018.02.015.

Listl S, Moeller J, Manski R. 2014. A multi-country comparison of reasons for dental nonattendance. Eur J Oral Sci. 122(1):62-69. doi:10.1111/eos.12096.

Murakami K, Aida J, Ohkubo T, Hashimoto H. 2014. Income-related inequalities in preventive and curative dental care use among working-age Japanese adults in urban areas: A cross-sectional study. BMC Oral Health. 14(1). doi:10.1186/1472-6831-14-117.

Nishide A, Fujita M, Sato Y, Nagashima K, Takahashi S, Hata A. 2017. Income-related inequalities in access to dental care services in Japan. Int J Environ Res Public Health. 14(5). doi:10.3390/ijerph14050524.

OECD. Health Care Resources: Dentist's Consultation. [accessed 2020 Apr 4]. https://stats.oecd.org/Index.aspx?DataSetCode=HEALTH_PROC.

OECD. 2011. Health at a Glance 2011: OECD Indicators. Paris. [accessed 2020 Mar 27]. http://dx.doi.org/10.1787/health_glance-2011-en.

OECD. 2019. Health at a Glance 2019: OECD Indicators. [accessed 2020 Apr 3]. https://www.oecd-ilibrary.org/social-issues-migration-health/health-at-a-glance-2019_4dd50c09-en.

Rozier RG, White BA, Slade GD. 2017. Trends in Oral Diseases in the U.S. Population. J Dent Educ. 81(8):eS97-eS109. doi:10.21815/JDE.017.016.

Rubin DB. 2004. Multiple imputation for nonresponse in surveys. John Wiley \& Sons.

Sakamoto H, Rahman MM, Nomura S, Okamoto E, Koike S, Yasunaga H, Kawakami N, Hashimoto H, Kondo N, Abe S, et al. 2018. Japan Health System Review. World Health Organization, Regional Office for South-East Asia.

Sheiham A, Steele J. 2001. Does the condition of the mouth and teeth affect the ability to eat certain foods, nutrient and dietary intake and nutritional status amongst older people? Public Health Nutr. 4(3):797-803. doi:10.1079/phn2000116.

Shigeoka H. 2014. The Effect of Cost Sharing on Utilization,Health,and Risk Protection. Am Econ Rev. 104(7):2152-2184.

Stock C, Jürges H, Shen J, Bozorgmehr K, Listl S. 2016. A comparison of tooth retention and replacement across 15 countries in the over-50s. Community Dent Oral Epidemiol. 44(3):223-231. doi:10.1111/cdoe.12209.

Thompson B, Cooney P, Lawrence H, Ravaghi V, Quiñonez C. 2014. Cost as a barrier to accessing 
dental care: findings from a Canadian population-based study. J Public Health Dent. 74(3):210-218. doi:10.1111/jphd.12048.

Vujicic M, Buchmueller T, Klein R. 2016. Dental Care Presents The Highest Level Of Financial Barriers, Compared To Other Types Of Health Care Services. Health Aff. 35(12):2176-2182. doi:10.1377/hlthaff.2016.0800.

Watt RG, Daly B, Allison P, Macpherson LMD, Venturelli R, Listl S, Weyant RJ, Mathur MR, Guarnizo-Herreño CC, Celeste RK, et al. 2019. Ending the neglect of global oral health: time for radical action. Lancet. 394(10194):261-272. doi:10.1016/S0140-6736(19)31133-X.

Watt RG, Heilmann A, Listl S, Peres MA. 2016. London Charter on Oral Health Inequalities. J Dent Res. 95(3):245-247. doi:10.1177/0022034515622198.

WHO. WHO | Universal coverage - three dimensions. [accessed 2020 Apr 3]. https://www.who.int/health_financing/strategy/dimensions/en/.

WHO. 2005. Sustainable health financing, universal coverage and social health insurance: World Health Assembly Resolution 58.33.

WHO. 2013. World health report 2013: Research for universal health coverage. https://apps.who.int/iris/bitstream/handle/10665/85761/9789240690837_eng.pdf?sequence=2.

Zaitsu T, Saito T, Kawaguchi Y. 2018. The Oral Healthcare System in Japan. Healthcare. 6(3):79. doi:10.3390/healthcare6030079.

\section{List of figure and table legends:}

Figure 1. Selection of the participants for the study

Figure 2. Shows discontinuity in the probability of dental visits during past 12 months before and after the discount eligibility (means of probability of dental attendance during past 12 months for each age are plotted with $2^{\text {nd }}$ order polynomial fit lines)

Table 1. Characteristics of the sample by discount eligibility $(\mathrm{N}=7,161)$

Table 2. Results of logistic regression models predicting dental visits during past 12 months (estimating the effect of JUHI discount eligibility): $\mathrm{N}=7,161$ 